\title{
A Machine Learning Approach to Speech Act Classification Using Function Words
}

\author{
James O'Shea, Zuhair Bandar and Keeley Crockett \\ Department of Computing and Mathematics \\ Manchester Metropolitan University \\ United Kingdom \\ \{z.bandar, k.crockett, j.d.oshea \}@mmu.ac.uk
}

\begin{abstract}
This paper presents a novel technique for the classification of sentences as Dialogue Acts, based on structural information contained in function words. It focuses on classifying questions or non-questions as a generally useful task in agent-based systems. The proposed technique extracts salient features by replacing function words with numeric tokens and replacing each content word with a standard numeric wildcard token. The Decision Tree, which is a well-established classification technique, has been chosen for this work. Experiments provide evidence of potential for highly effective classification, with a significant achievement on a challenging dataset, before any optimisation of feature extraction has taken place.
\end{abstract}

Keywords:- Dialogue Act, Speech Act, Classification, Semantic Similarity, Decision Tree.

\section{Introduction}

Dialogue Act (DA) classification is an established element of research in the field of Dialogue Management [1-6]. This work is motivated by the application of DA classification to natural language interaction with Dialogue Systems (DSs) [7] and Robots [8].

The majority of current dialogue systems use Pattern Matching (PM) or Natural Language Processing (NLP) to analyse and answer a user utterance. PM systems have been reported as the best for developing dialogue systems that seem to be coherent and intelligent to users [9]. They support scalability to large numbers of users because they do not require preprocessing stages, but are labour intensive and therefore costly to develop and maintain. NLP systems have a substantial theoretical basis but require a chain of computationally intensive and error-prone stages such as pos-tagging, syntactical repair and parsing. This rules them out for web-based systems that must service many users in real time.

Short Text Semantic Similarity (STSS) offers an alternative approach to PM and NLP. A user utterance (a unit of dialogue containing a communicative action [1]) is 
acquired as a Short Text (ST) and compared with a set of prototype STs. The ST with the highest similarity to the user utterance is taken to be its meaning.

DA theory separates the purpose of the utterance from its propositional content (i.e. what it is about). So DAs such as the question "Is the door shut?", the instruction "Shut the door!" and the assertion "The door is shut." can be derived from the propositional content the door is shut. We believe that classifying the Dialogue Acts (DAs) will be a crucial first step in measuring the semantic similarity between a pair of STs.

This paper investigates the hypothesis that DAs can be classified into different categories solely using function words. Machine classification of DAs has used various techniques including n-gram models [2], Bayesian networks [10] and Decision Trees (DTs) [3]. These approaches have achieved good results, but they use computationally intensive feature extraction which rules them out from future realtime applications.

In this paper 4 techniques are implemented, tested and compared. It is clear that the DT approach performs best, producing a Slim Function Word Classifier (SFWC) with high computational efficiency and high classification accuracy. We classify DAs at a coarse level of granularity as we believe distinguishing between questions, instructions and assertions will be most useful in practical DSs [11] and Robotics applications [8].

The rest of this paper is organised as follows: section 1 briefly reviews recent work on DA classification, section 2 provides an overview of DTs - in particular C4.5, section 3 outlines the experimental procedure, section 4 discusses the results and section 5 contains conclusions and recommendations for future work.

\section{Prior Work}

\subsection{Function Words}

Words in the English Language can be divided into Function Words (a closed class of structural words such as articles, prepositions, determiners etc.) and Content Words (the open classes of nouns, verbs, adjectives and adverbs). "Stop word" lists contain words with a high frequency of occurrence - mostly function words with a few high frequency content words mixed in. There is no definitive list of stop words, although one by van Rijsbergen [12], which is often cited contains 250 words. Others posted on the web contain over 300 [13]. A set of 264 function words was compiled for this paper by combining stop word lists, removing the content words and then adding lowfrequency function words from dictionaries.

Information Retrieval (IR) places a greater value on content words than function words in searching for documents. Spärck-Jones' [14] TD/IDF approach and Salton's [15] Vector Space Model increased the contribution of low frequency words to the similarity measurement. Latent Semantic Analysis (LSA) removes 439 stop words [16], having "little or no difference on the SVD solution." [17] from the submitted terms or documents before comparing them. This list has not been published. Citeseer also removes stop words prior to performing word frequency calculation [18].

The STASIS [19] STSS measure used function words as well as content words because they carry structural information which is useful in interpreting sentence 
meaning [19]. However, STASIS can only detect matches between identical pairs of function words in the two STs. An improved performance has also recently been achieved by an STSS algorithm which makes use of a corpus-based measure combined with string similarity, but filters out function words [20].

\subsection{Features For DA Classification}

The most common feature used for DA classification is the n-gram, a short string of words extracted from a marked up corpus [2, 4]. Large numbers of n-grams are generated by any real-world corpus so a predictive subset is used (in [3] sets of 10 or 300). N-grams have been used to code features for Hidden Markov Models [4]. Bigrams such as CanYou and IWant [10] have been used in Bayesian classifiers, which also combined them with the DAs of previous utterances in the dialogue [1].

Cue phrases have been used as features for a simple, efficient classifier described by Webb et al [2]. A cue phrase is a longer form of n-gram, selected on the basis of predictability for particular DA classes. In operation, the classifier examines an utterance for cue phrases and assigns the class of the highest predictive phrase it contains. This ignores other potentially valuable information in the utterance.

In the LSA approach to DA classification [5], the features are terms in a "query" vector. A cosine measure is used to determine the vector of the closest matching "document" and the DA type of the match is assigned to the query. LSA normally removes function words [17], but it is not specified if this is the case with DA classification.

Keizer et al [10] used 13 surface features to train DTs in a comparison with a Bayesian network. Features included length, various starting bigrams, presence of particular words, positive/negative references and presence of particular grammatical classes. Keizer's work is pertinent to the work in this paper because it classifies DAs into two classes, forward-looking functions (acts that have an effect on following dialogue) or backward-looking functions (that relate to previous dialogue).

The closest work to this paper [3] uses a decision tree trained with a mix of features including the presence of a question mark, a count of the occurrences of the word OR, utterance length, a bigram of the labels of the last two DAs, n-grams of pos-tags for the utterance, $\mathrm{n}$-grams of words in the utterance and the top 10 predictive individual words. A review by Verbree et al [3] also refers to the use of verb type and cites a number of uses of prosodic features.

Regardless of their performance, these features are complex and computationally intensive to extract, apart from Cue Phrases which may discard considerable useful information.

\subsection{The Slim Function Word Classifier for Dialogue Acts}

The Slim Function Word Classifier (SFWC) approach assumes that sufficient information is contained in the function words of an utterance to allow its DA to be classified. Each word in the utterance is replaced with a unique token and all content words replaced by the same wildcard. This performed efficiently with a lookup table.

After training a classifier on labeled data, new utterances can be coded with the same lookup table and classified. This study focuses on classifying Questions vs. 
Non-questions. However new classifiers can be developed for other DAs and the efficiency of this technique supports multi-classifier systems. In particular, DTs are easy to code in assembly language and would probably require the execution of less than 100 machine instructions to make a classification.

The novelty of this work is the simplicity and efficiency of the features, which supports scalability for applications with many users (e.g. the UK national flu service which received 9.3 million hits per hour on the first day of operation [21]).

\subsection{Evaluation Datasets}

The normal method of evaluating classifiers is to measure their Classification Accuracy (CA) on test data which was not used to train the classifier. Comparing DA classifiers is difficult as there is no standard dataset for developing and testing them. The different datasets used are tagged with different numbers and types of DA [3], ranging from 2 [10] to 232 different tags [5]. The sizes of the datasets also vary, from 223,000 utterances in the Switchboard set [2] to one of 81 utterances for a DT classifier [10]. Variety is also an issue. One dataset of 2794 DAs was drawn from only two humans [4] and may not generalize beyond these two people. One example of very good performance was a CA of $89.27 \%$ for a classifier with over 40 DA categories using a large training set [3]. Results can be variable however; another study [6] achieved $92.9 \%$ on suggestions but scored 0 on queries.

Classifying a single DA requires a relatively small dataset, so this study collected new material using web sources that cover a variety of topics, from a variety of people, to test our hypothesis.

\section{Classification Techniques}

Four techniques, Decision Trees, Naïve Bayes, Bayesian Classifiers and Multi-Layer Perceptrons (MLP) were investigated for the work in this paper. DT induction is a highly effective method of machine learning for classification. One of the most wellestablished algorithms is C4.5 [22]. DTs partition the sample space recursively and the outcome is a set of rules induced from a training set of example instances previously labeled with their classes. The chief advantage of DTs over other classifiers is that the rules "explain" how they reach their decisions and (combined with pruning) provide a greater insight into the problem domain.

The starting point of a DT is one node with all examples labeled as belonging to one class. A node is 'impure' if the examples reaching that node are not all in the same class. During training impure nodes are successively partitioned to create new, purer nodes. The final, leaf, nodes are labeled with the appropriate class. Impure leaves, which may occur if the attributes have been tested exhaustively without reaching purity, are labeled with the majority class. An alternative pruning technique, Minimum Number of Objects (MNO) removes leaves with fewer than a specified number of cases and re-distributes them amongst leaves at the level above in the tree.

Bayesian techniques, such as Naïve Bayes and the Bayesian Network have long been of interest to NLP researchers due to their statistical origins. The Naïve Bayes classifier uses information extracted from a set of example instances to produce a probability estimate of the class of a new instance, assuming statistical independence between the attributes to make the estimate[23]. The Bayesian Network is an 
alternative to decision trees, which uses a Directed Acyclic Graph structure[23]. This structure is less constrained in allowing linkage between nodes than a pure tree. Also, the node does not contain a splitting rule, rather it contains a probability distribution that is used to predict the class probability for a particular instance. The proposed advantage of the Bayesian Network over the DT is that it retains information that is lost due to splitting in DTs. However, the Bayesian network is also sensitive to missing values and this is an issue in handling STs of varying lengths.

The Multi-Layer Perceptron (MLP) is an Artificial Neural Network (ANN), modeled on the biological structure of the brain [24]. Inputs are fed through a network of simple processing units with weighted connections to produce (in this application) a classification output. The MLP is trained using backpropagation, which uses a training set to modify the weights until the performance is optimised. Of the 4 techniques, the MLP is the least transparent in explaining its decisions, however it has the benefit of robustness to noise and missing values.

\section{Experimental Procedure}

\subsection{Dataset}

In order to investigate the general hypothesis that a DT using function words can discriminate between a selected class of dialogue act and the remaining classes, the particular case of Questions vs. Non-questions was chosen. This was because prior empirical experience with DSs has shown this to be a non-trivial problem and because the task is appropriate to other agent-based applications such as robotics.

Current goal-oriented DSs engage in dialogue over the web in a manner similar to instant messaging. Utterances consist of Statement, Question and Instruction DAs. Most of the questions put to the agent are similar to those in FAQ lists, requesting definitions, clarifications etc. and this determined the dataset. 1,660 questions were collected from the highest user-rated FAQ lists from the Usenet news system. 2288 non-questions were collected from "blogs of note" commended blogs on blogspot.com. These were supplemented with other FAQs and Blogs obtained from the web so that questions were balanced by non-questions derived from the same context. Contexts were highly varied including "IRS", "Immunisation", "Tattoo" and "Gasoline." The majority of non-questions were assertions (statements, clarifications and answers to previous questions) with some instructions.

The FAQ questions are straightforward, starting with a limited set of words (e.g. What, How, Is, Can, If etc). The non questions rarely started with these words so a set of additional non-questions was synthesized (e.g. starting with which, acting as a pronoun) to increase the challenge. We have observed empirically that confusion of this type of utterance with questions reduces users' confidence in the agent. The approach of synthesising rare data is well-established [25]. Sometimes the synthetic non-questions are not valid sentences in their own right, but they would make sense as a terminating utterance in a dialogue sequence.

A set of $50 \%$ questions, 25\% straightforward non-questions and 25\% "synthetic" questions was randomly sampled from these collections (see table 1). For each category, a randomly selected sample larger than the target size was taken. The sentences were then pre-processed to restrict the length to a maximum of 25 words, 
expand contracted forms, tokenise them to extract features for DT construction and remove duplicates occurring due to loss of information in the tokenising process.

Table 1. Example training data

\begin{tabular}{|l|l|}
\hline Category & \multicolumn{1}{|c|}{ Example } \\
\hline FAQ question & Does wearing caps or hats contribute to hair loss? \\
\hline Blog non-question & $\begin{array}{l}\text { Sometimes the psoriasis treatment causes the hair } \\
\text { loss. }\end{array}$ \\
\hline Synthetic non-question & $\begin{array}{l}\text { Which in many cases can be cured with a simple } \\
\text { lotion. }\end{array}$ \\
\hline
\end{tabular}

Decision trees require fixed length records, so an upper limit of 25 words was set (determined empirically). The final proportions in the dataset were 591 questions and 615 non-questions.

\subsection{Preprocessing}

Expansion involved a simple table lookup to replace contracted forms such as Don't with their full forms, i.e. Do not. Some contractions are ambiguous e.g. She'd could be She would or She had. For this paper we took a brute force approach of replacing all the variants with a single form (e.g. ' $d$ forms with would). This is to avoid complex preprocessing as parsing-based disambiguation would increase the computational load greatly. Apostrophe usage in forming possessives was ignored and punctuation was stripped out as part of preprocessing. This means that the presence of a question mark - which has been used as a feature in some studies [3] was not used.

The sentences were then processed as follows. Words were successively looked up in a table of function words and numeric tokens (composed from the list described in 1.1). If the word was found it was replaced by the appropriate token (range $1-264$ ). In this experiment the tokens were allocated in ascending order to the alphabetically sorted list of words, so that 1 represents the word "a" and 264 represents "yourselves".

If the word was not found it was replaced by the token 0 (indicating a content word). If the sentence contained fewer than 25 words, all empty slots were filled with a "no word present" token given the value 300 (see table 2). This particular value was chosen so that it would be easy for the DT algorithm to partition it from the word tokens.

Table 2. Tokenisation of training data

\begin{tabular}{|l|l|}
\hline Question & does wearing caps or hats contribute to hair loss \\
\hline Tokenised & $56,0,0,156,0,0,212,0,0,300,300,300,300,300,300,300$, \\
form & $300,300,300,300,300,300,300,300,300$ \\
\hline
\end{tabular}




\subsection{Experiments}

\subsubsection{Choice of Classifier}

This section evaluates 4 well-established classifiers: Naïve Bayesian, Multi-Layer Perceptron, Bayesian Network and C4.5 DT. It also compares them with two standard baseline measures, ZeroR and OneR. Every result reported in this paper used 60-fold cross validation and was repeated 9 times so that each reported CA summarises 600 classifier construction runs. Each classifier was run using its default parameter settings in Weka. Results, reported in table 3, are recorded as percentage CA. All significance reporting in this paper uses the corrected re-sampled $t$-test with $\alpha=0.05$.

Table 3. Classification accuracies for baseline measures and popular classifiers

\begin{tabular}{|l|l|l|l|l|l|l|}
\hline Classifier & ZeroR & OneR & $\begin{array}{l}\text { Naïve } \\
\text { Bayes }\end{array}$ & MLP & $\begin{array}{l}\text { Bayes } \\
\text { Net }\end{array}$ & C4.5 \\
\hline CA & 50.99 & 82.06 & 55.98 & 69.14 & 77.87 & 88.73 \\
\hline
\end{tabular}

Each increase in CA across the AI classifiers is significant. All of the classifiers outperform the ZeroR baseline significantly. C4.5 also outperforms the OneR classifier significantly and consequently was selected for the remaining experiments. The high performance of DTs, combined with their efficiency and transparency was conclusive in their choice for further experiments. These were conducted to determine the best CA and the smallest tree size obtainable by pruning, using the two pruning techniques from section 2. DTs were built using the C4.5 (version 8) algorithm. Experiments $1 \& 2$ use Confidence Interval pruning and experiments $3 \& 4$ use the MNO method.

Tree sizes in the tables below are reported as minimum - maximum numbers of nodes. The baseline is always the CA obtained with confidence $=0.25$ and $\mathrm{MNO}=2$.

\subsubsection{Experiments 1 \& 2}

Experiment 1 examined the effect of pruning at a series of confidence levels running from the default value of 0.25 to 0.05 . Table 3 shows that increased pruning leads to a significant increase in CA at confidence $=0.2$. This reaches a maximum of 89.43 at confidence $=0.04$.

Table 3. Results of pruning in experiment 1

\begin{tabular}{|l|l|l|l|l|l|}
\hline Conf & 0.25 & 0.2 & 0.15 & 0.1 & 0.05 \\
\hline CA & 88.73 & 89.31 & 89.32 & 89.35 & 89.37 \\
\hline Tree Size & $89-147$ & $79-135$ & $79-131$ & $69-117$ & $59-101$ \\
\hline
\end{tabular}

Experiment 2 examined the effect of confidence pruning to produce the most compact, efficient tree that did not suffer a statistically significant loss of CA.

Experiment 1 was repeated, using pruning confidence intervals running from 0.0005 to 0.0001. At confidence $=0.0002$ the reduction in $\mathrm{CA}$ from the baseline was significant. Table 4 shows that pruning at confidence $=0.0003$ yields a good combination of tree size and CA. 
Table 4. Results of pruning in experiment 2

\begin{tabular}{|l|l|l|l|l|l|}
\hline Conf & 0.0005 & 0.0004 & 0.0003 & 0.0002 & 0.0001 \\
\hline CA & 88.48 & 88.25 & 87.74 & 85.29 & 84.21 \\
\hline Tree Size & $53-97$ & $51-95$ & $45-91$ & $43-87$ & $43-87$ \\
\hline
\end{tabular}

\subsubsection{Experiments $3 \& 4$}

Experiment 3 examined the effect of pruning at a series of MNO levels running from the default value of 2 to 20. Table 5 shows that increased pruning does not discover a significant increase in CA. At $\mathrm{MNO}=15$ the reduction in CA from the baseline was reported as significant.

Table 5. Results of pruning in experiment 3

\begin{tabular}{|l|l|l|l|l|l|}
\hline MNO & 2 & 5 & 10 & 15 & 20 \\
\hline CA & 88.73 & 88.97 & 88.29 & 85.83 & 83.64 \\
\hline Tree Size & $89-147$ & $59-99$ & $45-65$ & $31-57$ & $27-45$ \\
\hline
\end{tabular}

Experiment 4 examined the effect of MNO pruning to produce the most compact, efficient tree that did not suffer a statistically significant loss of CA. It repeated the process of experiment 3, using pruning MNO levels running from 10 to 14 instances. At $\mathrm{MNO}=14$ the reduction in CA from the baseline was significant. Table 6 shows that pruning at $\mathrm{MNO}=13$ yields a good combination of tree size and CA.

Table 6. Results of pruning in experiment 4

\begin{tabular}{|l|l|l|l|l|l|}
\hline MNO & 10 & 11 & 12 & 13 & 14 \\
\hline CA & 88.29 & 87.89 & 87.49 & 86.83 & 85.7 \\
\hline Tree Size & $45-65$ & $39-63$ & $41-59$ & $35-59$ & $33-61$ \\
\hline
\end{tabular}

\section{Discussion of Results}

The ZeroR baseline for CA is $51 \%$. Confidence level pruning achieves $89.43 \% \mathrm{CA}$ at 0.04 and MNO pruning achieved 88.97 at 5 instances per leaf. These results are good but it is difficult to compare them with other studies directly [3].

The average baseline tree size is 118 nodes. Pruning at confidence level 0.0003 reduces the average tree size to 47 nodes without a statistically significant reduction in CA. Pruning at $\mathrm{MNO}=13$ reduces the tree size to 46 nodes. The maximum depth of nesting of a 49 node tree $(\mathrm{MNO}=13)$ is 11 , so an efficient implementation would have a very low computational overhead.

\section{Conclusions and Future Work}

The results provide strong evidence to support the hypothesis that function words alone can discriminate between one major class of speech act (questions) and others (assertions, instructions etc.). Statistically significant results (using the corrected re- 
sampled $t$-test) for CA well above baseline have been obtained. The reduction in tree size from pruning provides further strong evidence that true generalisation is taking place. Function word features spanning a range from the $1^{\text {st }}$ to the $11^{\text {th }}$ word are being combined, so the technique is exploiting genuinely new information.

Future work will involve the following. Firstly, optimisation of feature extraction could yield a significant improvement in CA. For example, pronouns such as "I" (99), "me" (120) and "you" (260) are currently widely separated in the input space. Experiments will investigate whether clustering words by function reduces top-level splitting to make more information available for later stages in the tree.

Secondly, further classifiers will be developed to cover the full range of DAs. Collection of an Instruction / Non-instruction dataset, which is highly relevant to Robotics dialogue $[8,26]$ is already in progress.

Thirdly, the technique will be extended to cover Indirect DAs. Straightforward questions are often embedded in other text, e.g. a problem description posted in a forum followed by "can you help?" There will also be an extension to forms like "I don't know how to ...".

Finally, it has become clear that function words can perform multiple functions with varying degrees of likelihood. For example "Since" can be either a preposition or a conjunction. Fuzzy DTs and ANNs should improve performance by allowing function words to participate in different function sets with varying degrees of set membership.

\section{References}

1. Keizer, S.: A Bayesian Approach to Dialogue Act Classification. In: BI-DIALOG 2001 the 5th Workshop on Formal Semantics and Pragmatics of Dialogue, pp. 88--94. ZiF, Bielefeld (2001)

2. Webb, N., Hepple, M., Wilks, Y.: Dialogue Act Classification Based on Intra-Utterance Features. In AAAI 2005. AAAI Press, Pittsburgh, Pennsylvania (2005)

3. Verbree, D., Rienks, R., Heylen, D.: Dialogue-Act Tagging Using Smart Feature Selection; Results On Multiple Corpora. In IEEE Spoken Language Technology Workshop, pp. 70-73. IEEE Press, New York (2006)

4. Venkataraman, A., Stolcke A., Shriberg, E.: Automatic Dialog Act Labeling With Minimal Supervision. In 9th Australian International Conference on Speech Science and Technology (2002)

5. Serafin, R., Di Eugenio, B., Glass M.: Latent Semantic Analysis for dialogue act classification. In The 2003 Conference of the North American Chapter of the Association for Computational Linguistics on Human Language Technology (2003)

6. Wermter, S., Lochel, M.: Learning Dialog Act Processing. In COLING 1996, 16th International Conference on Computational Linguistics (1996)

7. Li, Y., Bandar, Z., McLean, D., O'Shea, J.: A method for measuring sentence similarity and its application to conversational agents. In The 17th International Florida Artificial Intelligence Research Society Conference (FLAIRS 2004), pp. 820--825. AAAI Press (2004)

8. Längle, T., Lüth, T., Stopp, E., Herzog, G., Kamstrup. G.: KANTRA - A Natural Language Interface for Intelligent Robots. In Intelligent Autonomous Systems (IAS 4), pp. 357--364. (1995)

9. Bickmore, T., Giorgino T.: Health dialog systems for patients and consumers. J Biomed Inform. 39(5), 556--571 (2006) 
10. Keizer, S., op den Akker R., Nijholt, A.: Dialogue Act Recognition with Bayesian Networks for Dutch Dialogues. In Third SIGdial Workshop on Discourse and Dialogue, pp. 88--94 (2002)

11. Crockett, K., Bandar, Z., O'Shea, J., McLean, D.: Bullying and Debt: Developing Novel Applications of Dialogue Systems. In Knowledge and Reasoning in Practical Dialogue Systems (IJCAI). IJCAI: Pasadena, CA (2009)

12. van Rijsbergen, C.: Information Retrieval. Butterworths, Boston (1980)

13. Sanderson, M., http://ftp.dcs.glasgow.ac.uk/idom/ir_resources/linguistic_utils/stop_words.

14. Spärck-Jones, K.: A Statistical Interpretation of Term Specificity and its Application in Retrieval. Journal of Documentation. 28, 11--21 (1972)

15. Salton, G., Wong, A., Yang, C.: A Vector Space Model for Automatic Indexing. Communications of the ACM. 18(11), 613--620 (1975)

16. Deerwester, S., Dumais, S., Furnas, G., Harshman, R., Landauer, T., Lochbaum, K., Streeter, L.: Computer information retrieval using Latent Semantic Structure. Bell Communications Research Inc. U.S.P. Office. (1989)

17. Deerwester, S., Dumais, S., Furnas, G., Landauer, T., Harshman, R.: Indexing by Latent Semantic Analysis. Journal of the American Society of Information Science. 41(6), 391-407 (1990)

18. Bollacker, K., Lawrence, S., Giles C.: CiteSeer: An Autonomous Web Agent for Automatic Retrieval and Identification of Interesting Publications. In 2nd International ACM Conference on Autonomous Agents, pp. 116--123. ACM Press (1998)

19. Li, Y., Bandar, Z., McLean, D., O'Shea, J.: Sentence Similarity Based on Semantic Nets and Corpus Statistics. IEEE Transactions on Knowledge and Data Engineering, 18(8) 1138-1150 (2006)

20. Islam, A., Inkpen D.: Semantic Text Similarity using Corpus-Based Word Similarity and String Similarity. ACM Transactions on Knowledge Discovery from Data. 2(2), 1-25 (2008)

21. BBC, http://news.bbc.co.uk/1/hi/health/8166444.stm.

22. Quinlan, J.: C4.5: programs for machine learning. Morgan Kaufmann Publishers, San Mateo, California (1993)

23. Witten, I., Eibe, F.: Data Mining: Practical Machine Learning Tools and Techniques. 2 ed. Elsevier, San Francisco (2005)

24. Aleksander, I., Morton H.: Introduction to Neural Computing. International Thomson Computer Press (1995)

25. Quinlan, J.: Induction of Decision Trees. Machine Learning. 1(1), 81--106 (1986)

26. Fong, T., Grange, S., Thorpe, C., Baur, C.: Multi-robot remote driving with collaborative control. In IEEE International Workshop on Robot-Human Interactive Communication. (2001) 